\title{
Analysis and Design on the Models of Quick Stabilized Tracing Platform
}

\author{
Zhifei Lv ${ }^{1, a}$, Zhenhong Zhu ${ }^{2, b}$ \\ ${ }^{1}$ School of Instrumentation Science and Optoelectronics Engineering, Beihang University, Beijing \\ 100191, China \\ ${ }^{2}$ Beijing Huahang Radio Measurement Research Institute, Beijing 100013, China \\ alzf248@buaa.edu.cn, bzhuzhenhong1986@163.com
}

Keywords: tracking and stability. control loop. correct network. Kalman

\begin{abstract}
Through mathematical analysis, the motor is established, and then the tracking stability control loop is set up. The control loop is designed to correct the network design and improve the stability and tracking performance. Research shows that through the stabilization loop lag correction network design, can effectively weaken the influence of perturbation. Through the PI design in angle tracking loop, angle tracking static error can be reduced when the sight angle is inputted in the velocity mode.
\end{abstract}

\section{Introduction}

The role of quick stabilized tracing platform is to insulate the influence of vector perturbation and target motion on aspect angle, and realize continuous and stable tracking of targets. In order to eliminate the disturbance on the effect of target tracking, tracking platform mostly adopts the optical lens (or antenna) servo tracking system based on rate gyro stabilization loop, its key technical indicators are tracking accuracy, disturbance isolation, tracking speed and system bandwidth, etc.

With the development of precision tracking technology, signal processing becomes more and more complex, resulting in the influence of signal processing delay on servo tracking system performance more and more obvious. The pure delay brings considerable phase lag to outer tracking loop of servo tracking system, which restricts the improvement of each indicator of the servo system.

For the servo tracking system with tracking angle error propagation delay, the scientific and technical workers have carried out related technical methods study on the tracking system accuracy.

The use of compound control, that is, while not affecting the stability of the original closed loop system $^{[1]}$, the target speed signal is introduced to feed forward to speed loop ${ }^{[2]}$, using advanced control to compensate the dynamic lag of the system, which can improve the tracking accuracy of the system. But in practical applications, the target speed signal can not be introduced into by differential links, while, the widely used is equivalent compound control technique.

Literature ${ }^{[3]}$ proposed the equivalent compound control method, in which, the target location information was composed by miss distance information and information encoder, then target speed information was gained by predictive filtering waves. Literature ${ }^{[4]}$ proposed a kind of finite memory least square filter method to predict parameters of target motion. It can estimate the motion parameters of the target at present or in the future, and minimize the mean square error of the prediction. However, in practical application, the method is not as good as simulation method. The reasons, on the one hand,the simulation is very difficult to simulate the impact of random noise; on the other hand, the miss distance delay of TV is not a fixed value, while the premise of finite memory least squares prediction is the same interval between each memory point.

In this paper, on the basis of establishing the tracking stability control loop, traditional correction method is adopted to improve the performance of the system as much as possible. After that, Kalman filtering is used to predict the sight angle information, considering the influence of system noise and observation noise, and, the prediction module is added into simulation model with angle error correlation processing delay, forming equivalent compound control, compared with traditional PID 
control, the proposed method can effectively improve the tracking accuracy of the system, and the prediction accuracy is higher, and the effect of compensation delay can be achieved.

\section{Torque motor model}

The mathematical modeling of the control system, as an important part of the control system, the motor model analysis needs to be carried out first ${ }^{[5]}$.

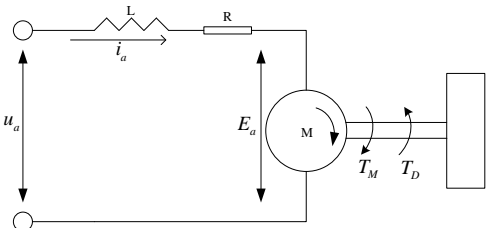

Fig.1 Schematic diagram of load torque motor

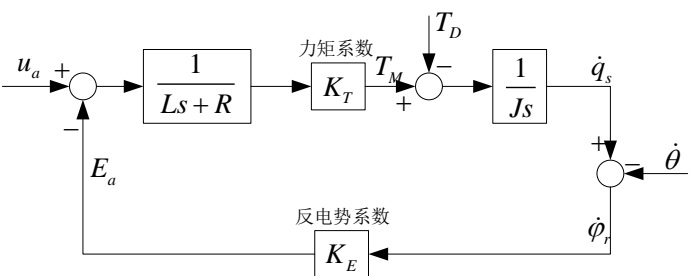

Fig.2 Control model of load torque motor

Figure 1 is the schematic diagram of load torque motor, where: $u_{a}$ is armature voltage; $i_{a}$ is armature current; and $\mathrm{R}$ is armature winding resistance.

$\mathrm{L}$ is armature winding inductance; $E_{a}$ is counter electromotive force; $T_{M}$ is motor output torque; and $T_{D}$ is resistance moment.

Based on figure 1,differential equation of motor dynamics can be gained as:

$$
\left\{\begin{array}{l}
u_{a}=R i_{a}+L \dot{i}_{a}+E_{a} \\
E_{a}=K_{E}\left(\dot{q}_{s}-\dot{\theta}\right) \\
T_{M}=K_{T} i_{a} \\
J \ddot{q}_{s}=T_{M}-T_{D}
\end{array}\right.
$$

Fig. 2 shows motor control block diagram which can be gained by differential equations, classical control theory knowledge.

The motor rotation angular velocity $\dot{q}_{s}$ is made as output, disturbance torque $T_{D}$ is 0 , the transfer function of torque motor model as described in the above figure can be gained ${ }^{[6]}$ :

$$
\frac{\dot{q}_{s}}{u_{a}}=\frac{1 / K_{E}}{\tau_{m} \tau_{e} s^{2}+\tau_{m} s+1}
$$

Where, $\tau_{m}=\frac{R J}{K_{E} K_{T}}$ is torque motor time constant,

$\tau_{e}=\frac{L}{R}$ is the electromagnetic time constant of torque motor,generally, $\tau_{m}>10 \tau_{e}$, the above formula can be written as:

$$
\frac{\dot{q}_{s}}{u_{a}}=\frac{1 / K_{E}}{\left(\tau_{m} s+1\right)\left(\tau_{e} s+1\right)}
$$

As $\tau_{e}$ is small, $1 / \tau_{e}$ exceeds the shear frequency of control system, which affects the control system at a very high frequency, in the analysis of low frequency control system, in order to simplify the analysis, the formula (2) can be further simplified as:

$$
\frac{\dot{q}_{s}}{u_{a}}=\frac{1 / K_{E}}{\tau_{m} s+1}
$$

\section{Tracking stable loop model}

Based on the motor model, the tracking loop and the stable loop model are established ${ }^{[7]}$.As shown in Fig.3: 


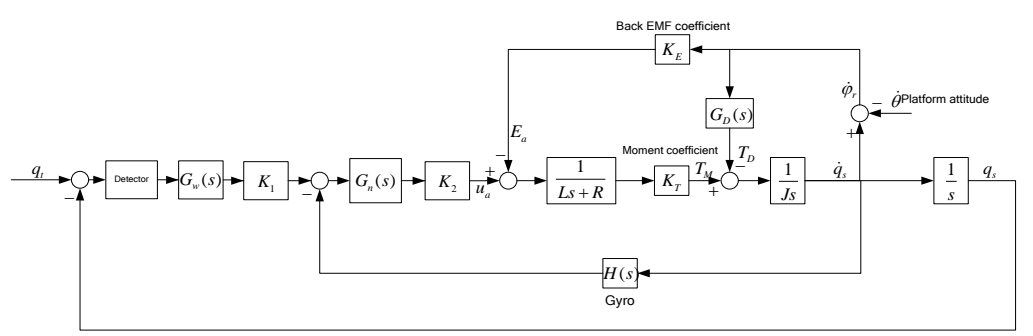

Fig.3 Tracking loop and stable loop model

Where, k is gain coefficient, $G_{w}(s)$ and $G_{n}(s)$ are respectively angle tracking loop and stable loop correction network.

In stable loop, isolation degree is an important index to judge the stability of the stability loop, it can be presented by the ratio of the rotation angular velocity and the angular velocity of the platform disturbance caused by the disturbance of the platform, here is the formula:

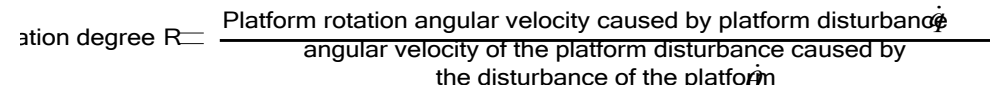

In the steady tracking state, the input signal and the feedback signal of the gyro are basically the same, because the gyro output is noisy, the input signal is used to represent the angular velocity of the motion of the platform. In the model, the transfer function from platform disturbance signal to the platform motion angular velocity output is:

$$
\dot{q}=-\frac{G_{D}(s) G_{w}(s)(L s+R) K_{1}+G_{w}(s) K_{T} K_{E} K_{1}}{s\left[(L s+R)\left(J s+G_{D}(s)\right)+K_{T} K_{E}+K_{2} K_{T} G_{n}(s) H\right]+K_{1} K_{2} G_{w}(s) G_{n}(s) K_{T}} \dot{\theta}
$$

The steady state value of the platform rotation angular velocity caused by the platform disturbance is:

$$
\left.\dot{q}\right|_{s=0}=-\left(\frac{G_{D}(s) R}{K_{2} G_{n}(s) K_{T}}+\frac{K_{E}}{K_{2} G_{n}(s)}\right) \dot{\theta}
$$

From formula (7), in order to reduce the degree of coupling of platform disturbance entering into the line of sight rate via counter electromotive force loop or disturbance torque loop, the design of $K_{2}$ and $G_{n}$ is required to make the system have a large enough gain in the working frequency band.

\section{Design of stability loop correction network}

Base and control object are connected by wire, bearing, when platform attitude changes, the relative motion between the control object and the base can interfere with the control object, affecting the spatial direction of the control object. In order to realize the stability of the control object points to the inertial space under platform attitude disturbance, the rate gyro feedback is used to compose stable loop, which is the inner loop of the angle tracking system.

Referring to the illustrated model 1 in figure 3, in order to facilitate the analysis of the stability loop, the circuit is analyzed separately. The stability loop can be reduced to the model as showed in Fig. $4^{[10]}$.

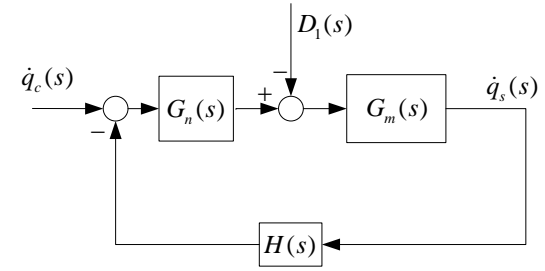

Fig.4 simplified model

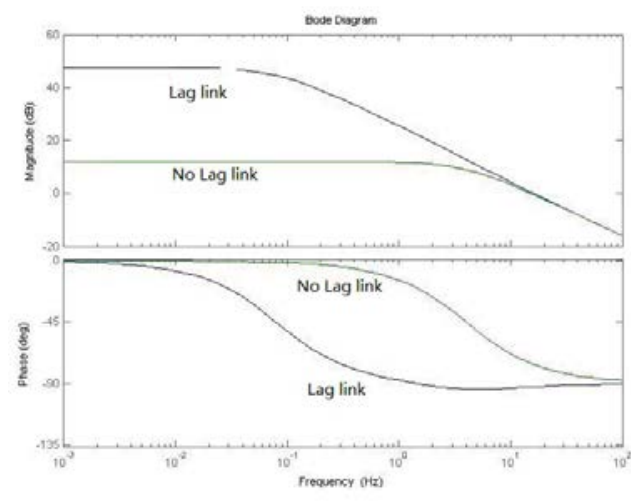

Fig.5 Comparison of open-loop Bode diagram 
Where, $G_{n}(s)$ is correction network, $G_{m}(s)$ integrates the power execution link of motor, power amplifier and transmission mechanism, $H(s)$ is gyro transfer function.

The transfer function of the disturbance torque is:

$$
\frac{\dot{q}_{s}(s)}{D_{1}(s)}=\frac{G_{m}(s)}{1+G_{m}(s) G_{n}(s) H(s)}
$$

Substitution with specific parameters to analyze.

Lag correction network:

$$
G_{n}(s)=\frac{\beta(T s+1)}{\beta T s+1}
$$

Where, $T=0.033, \beta=60$;

Power execution link:

$$
G_{m}(s)=\frac{4}{0.04 s+1}
$$

Gyroscope has a high bandwidth, for simple analysis, eliminating the dynamical part of the approximation to a proportional coefficient of 1 .

To draw the open loop frequency diagram of the network with or without lag correction, as shown in Fig.5.

According to the final value theorem, there is:

$$
\lim _{s \rightarrow 0} \frac{\dot{q}_{s}(s)}{D_{1}(s)}=\left\{\begin{array}{c}
\lim _{s \rightarrow 0} \frac{G_{m}(s)}{1+G_{n}(s) G_{m}(s) H(s)}=\frac{4}{1+240}=0.017 \text { Lag link } \\
\lim _{s \rightarrow 0} \frac{G_{m}(s)}{1+G_{m}(s) H(s)}=\frac{4}{1+4}=0.8 \text { No Lag link }
\end{array}\right.
$$

From Fig.5, the lag correction link increases the gain of the low frequency band, and has no influence on the high frequency characteristic of the stable loop, that is, it has no influence on the fast performance of the tracking control loop, and improves the ability of resisting interference of the stable loop. From formula (9), the existence of lag correction link greatly reduces the influence of interference on the angular velocity of the platform.

\section{Design of angle tracking loop correction network}

The main function of the angle tracking loop is to receive the angular error signal of the detector, control the motion of the detector, and realize the closed loop angle tracking of the target. Normally, angular tracking loop composed of detector and angle tracking loop can not meet the use requirements in stability, rapidity, system stability and anti-interference ability, therefore, we need to design the network to improve the transient stability of the angular tracking loop.

By referring to the model in Fig.3, $G_{w}(s)$ is the angle tracking loop correction network, usually the correction network has two forms: PI correction and lag correction ${ }^{[11]}$.

The simplest two expressions are taken:PI correction: $\frac{s+1}{s}$. lag correction: $\frac{5(0.2 s+1)}{s+1}$.

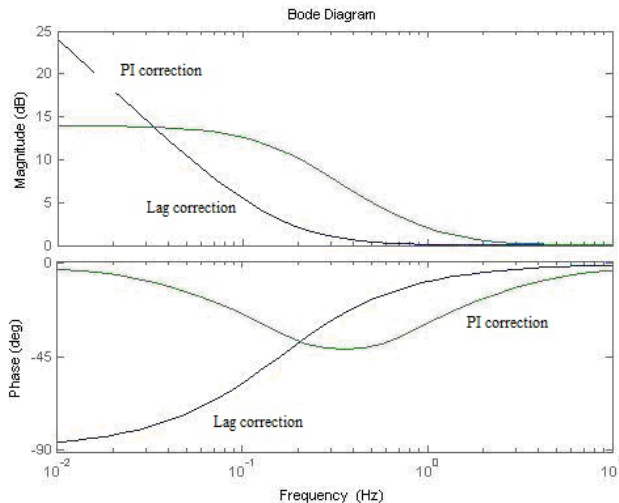

Fig.6 PI correction and lag correction Bode diagram

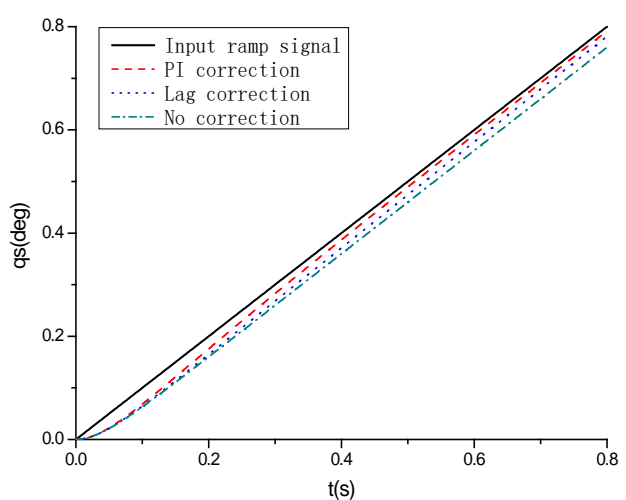

Fig.7 Time domain response of input of angular velocity 
Fig.6 shows that PI correction has higher gain in low frequency, and the lag correction has the increased gain in low frequency, but the increased number is determined by design value, which can not increase without limitation.

Fig.7 shows that if there is no calibration network, the curve of the point of view of the platform at the time can be gained when in line of sight speed signal input. And, when the correction network is introduced, the tracking error can be reduced, and the effect of the PI correction is better than that of the lag correction.

\section{Design and Simulation of tracking loop feed forward algorithm}

The line of sight velocity is estimated by the Kalman filtering algorithm, and the line of sight velocity information is fed forward to the stabilizing circuit.

The basic Kalman filtering equations are as follows:

One step prediction on status: $\hat{\mathrm{X}}_{\mathrm{k} / \mathrm{k}-1}=\Phi_{k \mathrm{k}-1} \hat{\mathrm{X}}_{\mathrm{k}-1 / \mathrm{k}-1}$

One step prediction mean square error: $\quad P_{k / k-1}=\Phi_{k / k-1} P_{k-1 / k-1} \Phi_{k / k-1}^{T}+Q_{k-1}$

Filter gain: $\mathrm{K}_{\mathrm{k}}=\mathrm{P}_{\mathrm{k} k \mathrm{k}-1} \mathrm{H}_{\mathrm{k}}^{\mathrm{T}}\left(\mathrm{H}_{\mathrm{k}} \mathrm{P}_{\mathrm{k} / \mathrm{k}-1} \mathrm{H}_{\mathrm{k}}{ }^{\mathrm{T}}+\mathrm{R}_{\mathrm{k}}\right)^{-1}$

state estimation: $\quad \hat{\mathrm{X}}_{\mathrm{k} / \mathrm{k}}=\hat{\mathrm{X}}_{\mathrm{k} k \mathrm{k}-1}+\mathrm{K}_{\mathrm{k}}\left(\mathrm{Z}_{\mathrm{k}}-\mathrm{H}_{\mathrm{k}} \hat{\mathrm{X}}_{\mathrm{k} / \mathrm{k}-1}\right)$

Estimated mean square error: $P_{k}=\left(I-K_{k / k} H_{k}\right) P_{k / k-1}$

Only given the $\hat{\mathrm{X}}_{0}$ and $\mathrm{P}_{0}$, the state estimation $\hat{\mathrm{X}}_{\mathrm{k}}$ at $\mathrm{K}$ time can be gained based on the measured vale $\mathrm{Z}_{\mathrm{k}}$ at $\mathrm{K}$ time.

The lag time of 2 sampling period miss distances exist in the tracking servo system, if the the state estimation $\hat{X}_{k}$ at $k$ time is gained by the $Z_{k-2}$ at $k-2$ time, then, the $\hat{X}_{k / k-1}$ is needed to be calculated based on the the $\mathrm{Z}_{\mathrm{k}-2}$ at $\mathrm{k}-2$ time.

$$
\begin{aligned}
& \hat{\mathbf{X}}_{k / k-1}=\boldsymbol{\Phi}_{k / k-1} \hat{\mathbf{X}}_{k-1 / k-1}=\boldsymbol{\Phi}_{k / k-1}\left[\hat{\mathbf{X}}_{k-1 / k-2}+\mathbf{K}_{k-1}\left(\mathbf{Z}_{k-1}-\mathbf{H}_{k-1} \hat{\mathbf{X}}_{k-1 / k-2}\right)\right]=\boldsymbol{\Phi}_{k / k-1}\left[\left(\mathbf{I}-\mathbf{K}_{k-1} \mathbf{H}_{k-1}\right) \hat{\mathbf{X}}_{k-1 / k-2}+\mathbf{K}_{k-1} \mathbf{Z}_{k-1}\right] \\
& =\boldsymbol{\Phi}_{k / k-1}\left[\left(\mathbf{I}-\mathbf{K}_{k-1} \mathbf{H}_{k-1}\right)\left(\boldsymbol{\Phi}_{k-1 / k-2} \hat{\mathbf{X}}_{k-2 / k-2}\right)+\mathbf{K}_{k-1} \mathbf{Z}_{k-1}\right]=\boldsymbol{\Phi}_{k / k-1}\left[\left(\mathbf{I}-\mathbf{K}_{k-1} \mathbf{H}_{k-1}\right)\left(\boldsymbol{\Phi}_{k-1 / k-2}\left(\hat{\mathbf{X}}_{k-2 / k-3}+\mathbf{K}_{k-2}\left(\mathbf{Z}_{k-2}-\mathbf{H}_{k-2} \hat{\mathbf{X}}_{k-2 / k-3}\right)\right)\right)+\mathbf{K}_{k-1} \mathbf{Z}_{k-1}\right]
\end{aligned}
$$

Where, filter gain $\mathbf{K}_{k-1}$ is affected by the state covariance $\mathbf{P}_{k-2}$ at $k-2$ time.

In the tracing system, the calculation process of state prediction $\hat{\mathbf{X}}_{k / k-1}^{\prime}$ at $\mathrm{k}$ time is :

$$
\hat{\mathbf{X}}_{k / k-1}^{\prime}=\boldsymbol{\Phi}_{k / k-1}\left[\left(\mathbf{I}-\mathbf{K}_{k-1}^{\prime} \mathbf{H}_{k-1}\right)\left(\boldsymbol{\Phi}_{k-1 / k-2} \hat{\mathbf{X}}_{k-2 / k-2}\right)+\mathbf{K}_{k-1}^{\prime} \mathbf{Z}_{k-1}\right]=\boldsymbol{\Phi}_{k / k-1}\left[\left(\mathbf{I}-\mathbf{K}_{k-1}^{\prime} \mathbf{H}_{k-1}\right)\left(\boldsymbol{\Phi}_{k-1 / k-2} \hat{\mathbf{X}}_{k-2 / k-3}\right)\right]+\boldsymbol{\Phi}_{k / k-1} \mathbf{K}_{k-1}^{\prime} \mathbf{Z}_{k-1}
$$

There, in the servo tracking system, if wanting $\mathrm{t}$ gain the state predictive value $\hat{\mathbf{X}}_{k^{\prime k-1}}$ at $\mathrm{k}$ time, the difference between formula (16) and (15) needs to be calculated, marked as $\delta \hat{\mathbf{X}}_{k}$. Here, based on the idea of approximate estimation of data delay, the approximate calculation of $\delta \hat{\mathbf{X}}_{k}$ is as formula (17), and the equation of state estimation (13) in basic Kalman filtering algorithm is replaced by formula (18), the remaining equations are invariant, and a new Kalman filtering algorithm with delay processing is obtained.

$$
\begin{gathered}
\delta \hat{\mathbf{X}}_{k}=\boldsymbol{\Phi}_{k / k-1}\left(\mathbf{I}-\mathbf{K}_{k-1} \mathbf{H}_{k-1}\right) \boldsymbol{\Phi}_{k-1 / k-2} \mathbf{K}_{k-2}\left(\mathbf{Z}_{k-2}-\mathbf{H}_{k-2} \hat{\mathbf{X}}_{k-2 / k-3}\right) \\
\hat{\mathbf{X}}_{k / k}=\hat{\mathbf{X}}_{k-1 / k-1}^{\prime}+\delta \hat{\mathbf{X}}_{k}
\end{gathered}
$$

But, as the state prediction $\hat{\mathbf{X}}_{k-1 / k-1}^{\prime}$ at k-1 time used in this state estimation equation of the algorithm, so, in the process of algorithm execution, the the state prediction $\hat{\mathbf{X}}_{k-1 / k-1}^{\prime}$ at k-1 time shall be calculated based on the theory of formula (17) and (18), the specific steps as :

$$
\begin{gathered}
\hat{\mathbf{X}}_{k-2 / k-2}=\hat{\mathbf{X}}_{k-2 / k-3}+\mathbf{K}_{k-2}\left(\mathbf{Z}_{k-2}-\mathbf{H}_{k-2} \hat{\mathbf{X}}_{k-2 / k-3}\right) \\
\hat{\mathbf{X}}_{k-1 / k-2}=\Phi_{k-1 / k-2} \hat{\mathbf{X}}_{k-2 / k-2} \\
\delta \hat{\mathbf{X}}_{k-1}=\boldsymbol{\Phi}_{k-1 / k-2}\left(\mathbf{I}-\mathbf{K}_{k-2} \mathbf{H}_{k-2}\right) \boldsymbol{\Phi}_{k-2 / k-3} \mathbf{K}_{k-3}\left(\mathbf{Z}_{k-3}-\mathbf{H}_{k-3} \hat{\mathbf{X}}_{k-3 / k-4}\right) \\
\hat{\mathbf{X}}_{k-1 / k-1}=\hat{\mathbf{X}}_{k-1 / k-2}+\delta \hat{\mathbf{X}}_{k-1}
\end{gathered}
$$




$$
\begin{gathered}
\hat{\mathbf{X}}_{k / k-1}=\Phi_{k / k-1} \hat{\mathbf{X}}_{k-1 / k-1} \\
\delta \hat{\mathbf{X}}_{k}=\boldsymbol{\Phi}_{k / k-1}\left(\mathbf{I}-\mathbf{K}_{k-1} \mathbf{H}_{k-1}\right) \boldsymbol{\Phi}_{k-1 / k-2} \mathbf{K}_{k-2}\left(\mathbf{Z}_{k-2}-\mathbf{H}_{k-2} \hat{\mathbf{X}}_{k-2 / k-3}\right) \\
\hat{\mathbf{X}}_{k / k}=\hat{\mathbf{X}}_{k / k-1}+\delta \hat{\mathbf{X}}_{k}
\end{gathered}
$$

That is, first, the state value of $\hat{\mathbf{X}}_{k-2 / k-2}$ is predicted based on the measured value $\mathbf{Z}_{k-2}$ at k-2 time, then, according to formula (11) (12), and (14), recursive to calculate filtering gain of $\mathbf{K}_{k-1}, \mathbf{K}_{k-2}$, $\mathbf{K}_{k-3}$, and by (10) $\sim(12)$, the state value $\hat{\mathbf{X}}_{k-1 / k-1}$ at k-1 time is predicted, then predicting the state value $\hat{\mathbf{X}}_{k / k}$ at $\mathrm{k}$ time. But, i,n each recursive calculation, the filtering gain, mean square error, $\hat{\mathbf{X}}_{k-3 / k-4}$ and $\mathbf{Z}_{k-3}$ shall be saved.

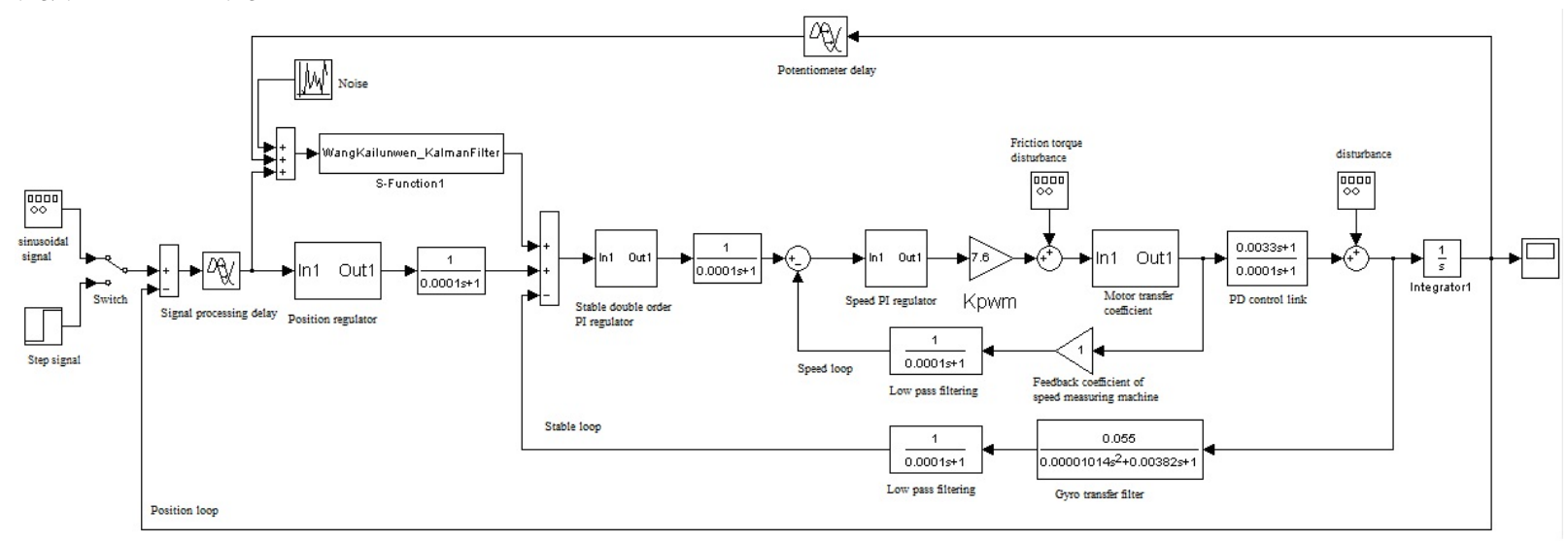

Fig.8 Simulation model of compound control based on Kalman

Building simulation model according to the previous introduction, as Fig.8.

Input signal:

Equivalent sinusoidal target: $\gamma(t)=60^{\circ} \sin (0.573 t)$. Step signal: $\gamma(t)=1^{\circ}$.

In order to show the simulation results more intuitive Kalman prediction filter,composite control results without delay, traditional PID simulation results and the equivalent compound control results from the Kalman predictive filter are respectively given, and merging the results of the 3 sets of experiments into a graph, as shown in Fig.9,10.

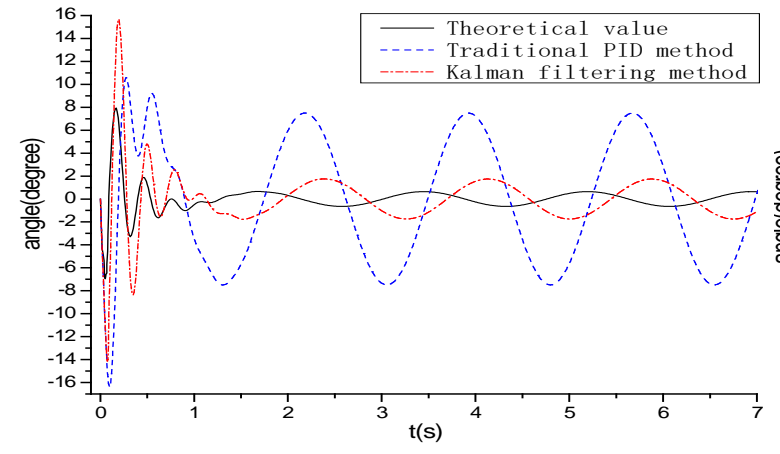

Fig.9 Equivalent sinusoidal target tracking error contrast curve

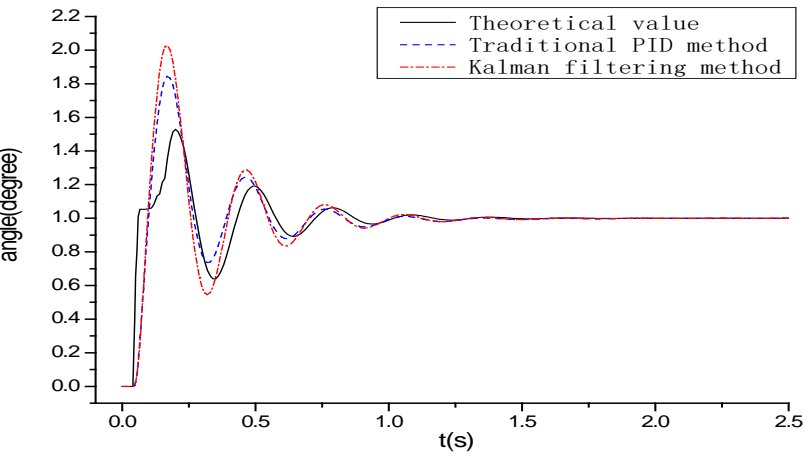

Fig.10 Step response contrast curve

From the simulation results, the use of predictive filtering can obviously improve the tracking precision of servo tracking system, and its effect of reducing steady state tracking error of tracking system is better than the traditional PID regulation. But, from the step response curve of the system, it is not difficult to find out the overshoot of the system using the predictive filter method is larger. For tracking platform system with larger field range, Kalman prediction filter can be used in the whole process, but for small field of view, such as television or photoelectric, the overshoot increase is easy to cause the target to fly out of the field of view to cause the tracking failure. Therefore, the method is not suitable for the initial stage of the capture target of the television or the photoelectric seeker, but suitable for the stable tracking stage. 


\section{Conclusion}

Through mathematical analysis, the mathematical model of the motor is established, and the tracking stable platform model is set up to provide the model foundation for the performance design of the control loop. The stable loop correction network design is made, lag correction network is used to make the system high frequency characteristic unchanged, and the rapidity is not affected; Low frequency gain enhancement, anti jamming ability enhancement, which can effectively reduce the influence of platform disturbance on the direction of the platform. PI calibration design is carried out for angle tracking loop, its characteristics of high and low frequency gain can greatly improve the open loop low frequency gain of angle tracking loop, which is advantageous to reduce the steady state error of the system, reduce the tracking error of the target, and reduce the loss of the target tracking. The analysis and design of the model can provide a theoretical basis and design reference for the practical engineering problems. At last, a Kalman filtering algorithm is designed to compensate the delay of signal processing, by simulation, it is proved that it can realize the prediction of the line of sight angle information, which is better than the traditional PID control in improving the tracking accuracy of the system, which can achieve the role of compensation delay.

\section{References}

[1] Jiaguang Ma, The basic problems of acquisition tracking and pointing system[J].Optical engineering,1989, 81( 3) : 1- 42.

[2] Jianli Wang.Research on video tracking and capture fast moving target of photoelectric theodolite[D].CHangchun: Changchun Institute of Optics and Fine Mechanics and Physics, 2002.

[3] Jiaguang Ma.ATP photoelectric acquisition tracking and pointing technology translation Anthology (second episodes)[M].Institute of optoelectronic technology, Chinese Academy of Sciences, 1995.

[4] Shaoyong Wu. Research on dynamic high type control method[D].Undergraduate thesis of University of Science \& Technology China,2004.

[5] Downs J, Smith S, Schw ickert J, et al . High performance gimbal control for self protection weapon systems [C] . Florida: SPIE, 1998.

[6] Crawthrop P. J, Khnrbouch M. Two-loop Cascade Control[C]. IEE Proceedings,1988, 135(3,): 232-238.

[7] Claudio Maffezzoni,Nicola Schiavoni,Gianni Ferretti . Robust Design of Cascade Control[C]. IEEEConlxol Systems Magazine, 1990, 10(1): 21-25.

[8] Yingying Cui, Qunli Xia, Zaikang Qi.Research on the isolation degree model of seeker stabilized platform[J].Journal of missile and guidance ,2006(5): 22-25

[9] Congjun Zhang.Design and analysis of the control loop of seeker stabilized platform[J].Journal of missile and guidance,2009(5): 46-48

[10] Wu Ye, Weifeng Hu, Meijian Xu.Linear isolation degree of seeker stabilized platform and its improving method[J].Guidance and Fuze,2011(1): 1-5

[11] Zhenhong Zhu, Qunli Xia, Chuntao Cai.Research on decoupling and line of sight rate extraction algorithm for phased array radar[J].Tactical missile technology,2013（5） : 70-75

[12] Fugui Li, Qunli Xia,Zaikang Qi,Influence of parasitic loop and identification correction of all Strapdown Seeker[J].System engineering and electronic technology,2013 (8) : 1717-1721 